\title{
Controlling the Perceived Vibrational Frequency and Amplitude of a Voice-Coil-Type Tactor
}

\author{
Masataka Niwa ${ }^{1,2 *}$ Haruo Noma ${ }^{1 *}$ Yasuyuki Yanagida ${ }^{3,1^{* *}}$ Kenichi Hosaka ${ }^{\text {* }} \quad$ Robert W. Lindeman ${ }^{4,1^{* \star *}}$ \\ ${ }^{1}$ ATR Media Information Science Laboratories \\ ${ }^{2}$ Graduate School of Information Science and Technology, Osaka Institute of Technology \\ ${ }^{3}$ Faculty of Science and Technology, Meijo University \\ ${ }^{4}$ Dept. of Computer Science, Worcester Polytechnic Institute
}

\begin{abstract}
When designing a vibrotactile information display, it is important to present correct, stable stimulation. However, the mechanical stability of the vibration is affected by many conditions such as how the vibrators are attached to the person, where they are attached, and other factors. In order to maintain the target frequency and amplitude of the vibration independent of the affective factors, we propose a feedback method for vibrotactile displays.
\end{abstract}

CR Categories: H.5 [Information Interface and Presentation]: User Interfaces-Haptic I/O

Keywords: vibrator, tactor, vibrotactile, haptic sensation, vibrotactile feedback

\section{INTRODUCTION}

Vibrotactile displays are used widely in many modern devices. The most common example is the vibrating motor of a cellular phone. In many cases, tactors, the devices that produce the vibration, are run with only a binary state, ON or OFF [1], because the mechanical stability of the vibration is affected by many conditions such as the orientation of the device, the movement of the device in space, and other factors. Most ongoing research, however, has not taken this sufficiently into account. If these factors can be ignored, and a tactor can be controlled quantitatively, there is a greater possibility of effective information display. Therefore, the purpose of this research is to maintain a precise vibration state for the tactor, regardless of outside forces.

\section{Perceived Vibration}

We can define a transfer function $J$ that maps from an input state $t$ to an output state $s$, where $t$ is the control signal to the vibrotactile device and $s$ is the perceived vibration by the user (Figure 1(a)). When we first started using DC-motor-type vibrators as tactors, we found that the oscillating frequency and amplitude of this type of device were affected by such factors as the method used to mount them on the person, and the orientation of the device in relation to the gravity vector, even when the electric signal was kept constant. Considering these effects, we can decompose $J$ into

*e-mail: \{m-niwa, noma, hosaka $\}$ atr.jp

**e-mail: yanagida@ccmfs.meijo-u.ac.jp

***e-mail: gogo@wpi.edu

Symposium on Haptic Interfaces for

Virtual Environment and Teleoperator Systems 2006

March 25 - 26, Alexandria, Virginia, USA

1-4244-0226-3/06/\$20.00 O2006 IEEE (a)

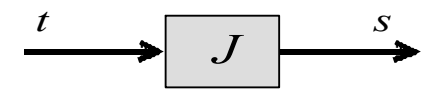

(b)

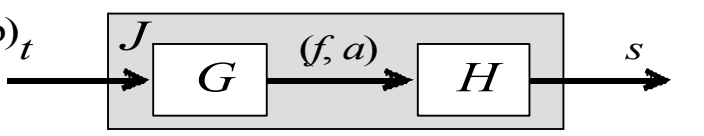

Figure 1. Transfer functions for vibrotactile stimuli (a) modeled as a single function, and (b) modeled as a series of functions affected by various factors.

two components, $G$ and $H$, and model the process of how this type of tactor makes us perceive tactile sensations as shown in Figure $1(\mathrm{~b})$.

The input state variable for the transfer function $G$ is the electric signal for the vibrator, and the output state variable is the vibration characteristics, such as frequency $f$ and amplitude $a$. Variable factors for the transfer function $G$ are mainly the electrical and mechanical conditions of the vibrator: how the vibrators are mounted. These factors affect the actual magnitude of tactor vibration as well as cause a disturbance in the transfer function $\mathrm{G}$. The output state variable for the transfer function $H$ is the tactile sensation $s$ : how the user feels the vibration. The transfer function $H$ is related to the method of mounting, as well as the mental and physical condition of the user. As long as a constant electrical signal is applied, DC-motor-type vibrators work at a constant frequency and amplitude when rigidly fastened to a surface, such as a tabletop. In other words, the state of vibration corresponds one-to-one to the state of the applied electric signal, as illustrated in Figure 1(a). Most of the tactile displays using vibrotactile tactors have been designed with this one-to-one mapping as a presupposition; they model the transfer functions $G$ and $H$ as a single transfer function, record the correspondence relation of output $s$ to a certain input $t$ beforehand, and expect that this relation will always hold. However, as described in our model, dynamic changes in the environment make the output of $\mathrm{H}$ vary constantly due to mechanical factors. Therefore, there is the possibility that a user will receive a different tactile sensation when his or her posture changes, even when $t$ is held constant.

In our previous work, we manipulated only the frequency of a DC-motor-type tactor [4], as frequency and amplitude are mechanically coupled in these devices. In this paper, we use a voice-coil-type tactor (MMA-33 by NEC Tokin Corporation), which allows us to control both the frequency and the amplitude independently.

\section{DESIGN}

Our solution utilizes a piezoelectric film sensor (Figure. 2, SDT1$028 \mathrm{~K}$ by Tokyo Sensor Co.,Ltd.) that measures the vibration 
status and uses a feedback-loop circuit to dynamically adjust the input to the tactor driver to maintain the target frequency and amplitude. The feedback loop is carried at $200 \mathrm{~Hz}$, and a PI (Proportional and Integral) controller is employed.

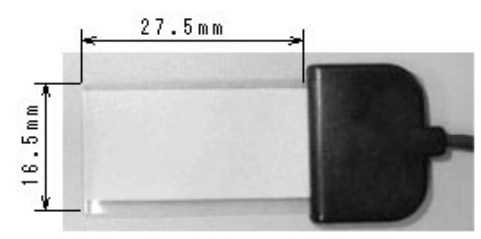

Figure 2. Piezoelectric film sensor.

We monitored the vibration of the tactor in both loaded and unloaded conditions to verify that our feedback control functions correctly. The loaded condition was achieved by affixing the tactor/sensor package to the arm, wrapping it with a bloodpressure cuff, and varying the amount of inflation of the cuff. The vibration was then triggered, and the cuff pressure was switched between $0 \mathrm{~Pa}$ to $5.3 \times 10^{3} \mathrm{~Pa}$. Based on results from previous experiments, the target frequency was set to $148 \mathrm{~Hz}$ and the target amplitude was set to $2.4 \mathrm{~V}$.

The results are shown in Figure 3. The horizontal axis represents elapsed time, and the vertical axis represents amplitude. The interval marked "Increase" in the figure means that the cuff pressure is being increased. When our feedback method is not active, the amplitude varies as the cuff pressure varies. However, when our method is active, amplitude voltage produced by the sensor converges on the target amplitude. The frequency is not affected by changes in cuff pressure, so frequency control and feedback are not necessary for voice-coil-type tactors. Therefore the frequency and the amplitude of the voice-coil-type vibrator can be controlled by using this method. This method controls the output state of transfer function $G$ as shown in Figure 4. The he transfer function $H$ is thus not affected by the feedback control.
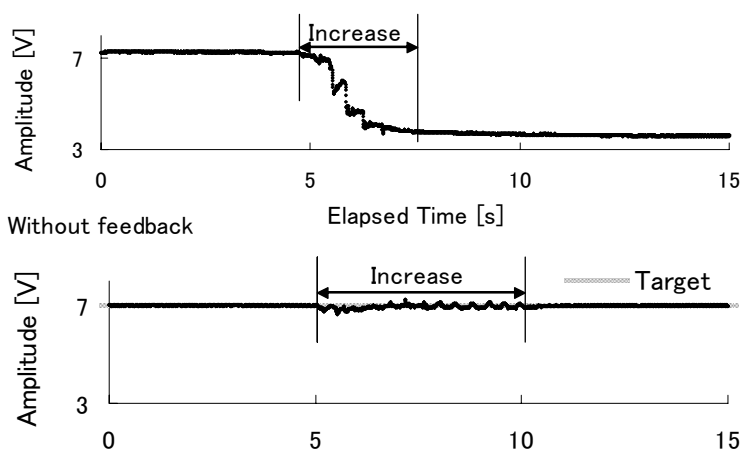

With feedback

Elapsed Time [s]

Figure 3. The amplitude while the vibrator is pressed without and with feedback.

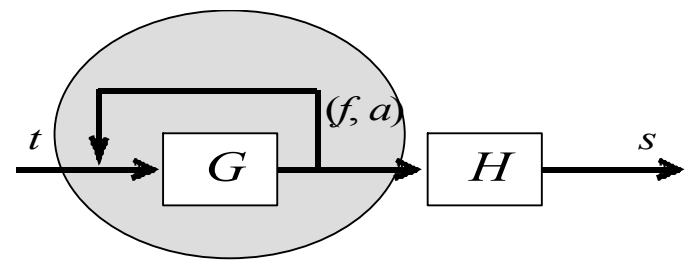

Figure 4. Controlling the output state of transfer function $G$.

\section{EMPIRICAL STUDY}

The tactor was then started and pressure was applied to the arm by inflating the cuff. After the change in pressure, the subject was asked if he or she felt a difference in the intensity of the tactor. The subject was given a choice of increased or decreased intensity in a forced-choice test. Each of the 20 subjects was given 40 trials, with half the trials starting with the cuff inflated and half starting with the cuff deflated. The feedback system was only activated in half of the trials.

The results are shown in Figure 5. The horizontal axis indicates the results obtained with the feedback OFF or ON, and the vertical axis represents the rate at which subjects answered that the perceived vibration was strongest. The results show that, in the presence of our feedback method, subjects identified a significantly larger number of trials correctly than when our feedback method was deactivated. We found a $5 \%$ chi-square statistical difference between Feedback OFF and Feedback ON. This indicates the usefulness of such a feedback system on influencing perception.

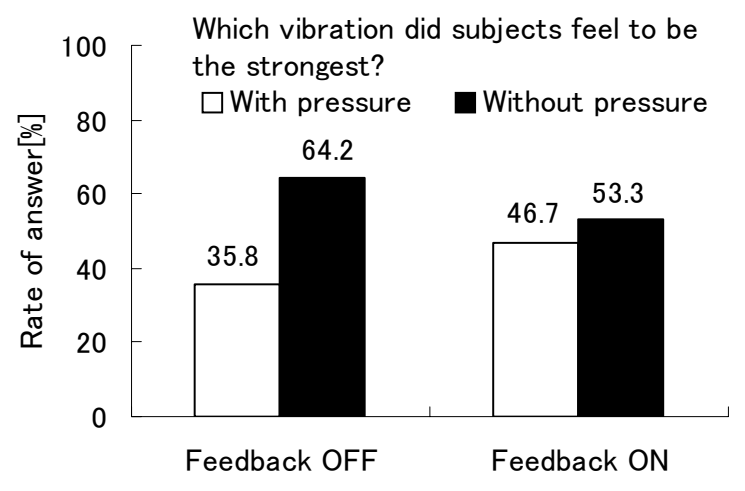

Figure 5. User-study results.

\section{CONCLUSION}

The mechanical stability of vibration is affected by many conditions. When designing a vibrotactile information display, it is important to present correct and repeatable stimuli, even in the presence of dynamic changes to the environment. This system can be used as a vibrotactile calibrator. If tactile sensation needs to be controlled, additional feedback is necessary.

\section{ACKNOWLEDGMENTS}

This research was supported in part by the National Institute of Information and Communications Technology in Japan.

\section{REFERENCE}

[1] A. Rupert, "An instrumentation solution for reducing spatial disorientation mishaps", IEEE Eng. in Med. and Bio. 2000, pp. 7180, March-April 2000.

[2] L.A. Jones, M. Nakamura and B. Lockyer, "Development of a Tactile Vest", Proceedings of 12th International Symposium on Haptic Interfaces for Virtual Environment and Teleoperator Systems, pp. 82-89, March 2004.

[3] R.W. Lindeman and Y. Yanagida, "Empirical Studies For Effective Near-Field Haptics in Virtual Environments", Proc. of IEEE Virtual Reality 2003, Los Angeles, U.S.A, pp. 287-288, March 2003.

[4] J. Cohen, M. Niwa, R.W. Lindeman, H. Noma, Y. Yanagida, and K. Hosaka, "A Closed-Loop Tactor Frequency Control System for Vibrotactile Feedback", ACM CHI2005 Extended Abstracts, Portland, Oregon, USA, pp. 1296-1299, April 2005. 